\title{
Approximation hardness of edge dominating set problems*
}

\author{
Miroslav Chlebík ${ }^{\dagger}$ \& Janka Chlebíková ${ }^{\prime}$
}

\begin{abstract}
We provide the first interesting explicit lower bounds on efficient approximability for two closely related optimization problems in graphs, Minimum Edge Dominating Set and Minimum Maximal Matching. We show that it is NP-hard to approximate the solution of both problems to within any constant factor smaller than $\frac{7}{6}$. The result extends with negligible loss to bounded degree graphs and to everywhere dense graphs.
\end{abstract}

Keywords: minimum edge dominating set, minimum maximal matching, approximation lower bound, bounded degree graphs, everywhere dense graphs

\section{Introduction}

An edge dominating set for a simple graph $G=(V, E)$ is a subset $D$ of $E$ such that for all $e \in E \backslash D$ there is an edge $f \in D$ such that $e$ and $f$ are adjacent. The Minimum Edge Dominating Set problem (shortly, Min-Eds) asks to find an edge dominating set of minimum cardinality, eds $(G)$ (resp. minimum total weight in weighted case). The decision version of MiN-EDS was shown to be NP-complete even for planar (or bipartite) graphs of maximum degree 3 by Yannakakis and Gavril [18]. Later Horton and Kilakos extended their results showing NP-completeness also for planar bipartite graphs, line graphs, total graphs, perfect claw-free graphs, and planar 3-regular graphs [13]. On the other hand, the problem admits polynomial-time approximation scheme (PTAS) for planar graphs [1], or for $\lambda$-precision unit disk graphs [14]. Some special classes of graphs for which the problem is polynomially solvable have been discovered, e.g. trees [15], claw-free chordal graphs, locally connected claw-free graphs, line graphs of total graphs, line graphs of chordal graphs [13], bipartite permutation graphs, cotriangulated graphs [17].

\footnotetext{
* An extended abstract of this paper was accepted at the 14th Annual International Symposium on Algorithms and Computation, ISAAC 2003.

${ }^{\dagger}$ Max Planck Institute for Mathematics in the Sciences, Inselstraße 22-26, D-04103 Leipzig, Germany, chlebik@mis.mpg.de

${ }^{\ddagger}$ Department of Informatics Education, Faculty of Mathematics, Physics, and Informatics, Comenius University, 84248 Bratislava, Slovakia, chlebikova@fmph.uniba.sk
} 
An edge dominating set of minimum cardinality has close relationship with minimum maximal matchings. A matching in a graph $G=(V, E)$ is a subset of edges $E$ with no shared end nodes. A matching is maximal if no other matching properly contains it (or, equivalently, if a matching is an edge dominating set). The Minimum Maximal Matching problem (shortly, Min-Maxl-Match) asks to find a maximal matching of minimum cardinality.

The fact that there are much more edge dominating sets than maximal matchings in a graph does not distinguish the optimization problems Min-EDS and Min-Maxl-Match significantly. In fact, the minimum cardinality of edge dominating sets in a graph is achieved also on its maximal matchings. Even more interestingly, there is a simple polynomial-time algorithm to transform any given edge dominating set $D$ in $G$ to a maximal matching $M$ in $G$ such that $|M| \leq|D|$ (see, e.g. [18]). Due to this fact the problems Min-Eds and MinMAXL-MATCH are equivalent. Any polynomial time $\rho$-approximation algorithm for Min-EDs can be easily transformed to the one for Min-MAXL-MATCH with the same performance ratio; the converse relation being trivial. It is easy to observe that no matching in a graph can be more than twice larger than any maximal matching. Therefore constructing any maximal matching (which is possible in $O(|E|)$ time) suffices to approximate the problems MiN-EDS and Min-MAXL-MATCH to within a factor 2. Recently, also weighted version of Min-EDS was shown to be approximable efficiently to within a factor 2 ([10], $[16])$.

For a graph $G=(V, E)$ a subset $C \subseteq V$ is a node cover, if every $e \in E$ is incident to some node in $C$. The Minimum Node Cover problem (shortly, MIN-NC) asks to find a node cover of minimum cardinality, $n c(G)$. It is quite straightforward via simple reduction (see [2]) that weighted MiN-EDS is at least as hard to approximate as MiN-NC. Hence any inapproximability result for Min-NC applies directly to weighted Min-EDS. In particular, the result of Dinur and Safra [7] implies that it is NP-hard to approximate weighted MiNEDs to within any constant factor smaller than $10 \sqrt{5}-21 \approx 1.36067$.

For (unweighted) Min-EDs, or equivalently, Min-MAXL-MATCH, the gap is much wider between the upper bound 2 and the known lower bound on approximability. The transformation of Yannakakis and Gavril ([18]) showing NP-completeness of Min-MAXL-MATCH reducing 3-Min-NC (the restriction of Min-NC to 3-regular graphs) to it, may be regarded as an $L$-reduction and hence gives APX-completeness for problems we are interested in. This implies NP-hardness to approximate Min-MAXL-MATCH to within a factor $1+\delta$ for some $\delta>0$. But lower estimates on $\delta$ obtained from inapproximability results for 3 -Min-NC, and from parameters of that $L$-reductions are only about $\frac{1}{500}$.

In Section 3 we prove that it is NP-hard to approximate the problem MiNEDs (and hence also Min-MAXL-MATCH) to within any factor smaller than $\frac{7}{6}$. We provide similar lower bound $\frac{7}{6}-\frac{24 \log B}{B}$ for graphs of the maximum degree $B, B \geq 462$, and $\frac{7+\theta}{6+2 \theta}$ for everywhere $\theta$-dense graphs. In Section 4 we present some results for very small degree instances: lower bound $1+\frac{1}{487}$ for graphs with maximum degree 3 and slightly better lower bounds for sparse bipartite graphs 
with all nodes but one of degree $B$. Namely we prove NP-hardness factors of $1+\frac{1}{390}$ and $1+\frac{1}{250}$ for $B=4$ and $B=5$, respectively.

\section{Preliminaries}

It is easy to see that a set of edges $M \subseteq E$ is an edge dominating set of $G=(V, E)$ if and only if $V(M)$, the set of end nodes of edges from $M$, is a node cover of $G$. Hence any edge dominating set (in particular, any maximal matching) $M$ of $G$ satisfies

$$
2|M| \geq|V(M)| \geq n c(G) .
$$

Consequently, $n c(G) \leq 2 e d s(G)$ holds for every graph $G$. Those graphs $G$, for which bound $n c(G)=2 e d s(G)$ is achieved, will be of our main interest in what follows.

Let us denote by $\mathcal{G}$ the class of graphs $G=(V, E)$ for which a minimum cardinality node cover $C \subseteq V$ of $G$ exists such that the subgraph induced by $C$ has a perfect matching, that is a matching $M$ with $V(M)=C$. Clearly, any perfect matching $M$ in the graph induced by $C$ is a maximal matching of $G$, as its node set $V(M)$ is a node cover of $G$. Moreover, as $2|M|=|V(M)|=|C|=$ $n c(G), M$ is a minimum maximal matching of $G$ due to (1). Hence, we have just verified that $n c(G)=2 e d s(G)$ for every $G \in \mathcal{G}$. Due to this simple relation between eds and $n c$, our goal is to prove suitable NP-hard gap results for the Min-NC problem restricted to $\mathcal{G}$. In fact, we will deal with even more restricted class $\mathcal{G}_{0} \subseteq \mathcal{G}$ of graphs $G=(V, E)$ for which every minimal (inclusionwise) node cover $C \subseteq V$ of $G$ induces the subgraph with a perfect matching. For this purpose we have to show first that $\mathcal{G}_{0}$ is rich enough.

We start with some combinatorial notions and structural properties ensuring that a graph belongs to $\mathcal{G}_{0}$.

Definition 1 A graph $G[s]=(V[s], E[s]$ ) is an s-padding (s being a positive integer) of a graph $G=(V, E)$, if $G[s]$ is obtained from $G$ by replacing every node $v \in V$ by a set $v[s]:=\left\{v_{1}, v_{2}, \ldots, v_{s}\right\}$ of $s$ distinct copies of $v$, and taking $E[s]:=\left\{\left\{u_{i}, v_{j}\right\}:\{u, v\} \in E, i, j \in\{1,2, \ldots, s\}\right\}$.

This graph operation has been frequently used and many of its basic properties are well known. Clearly, whenever $C \subseteq V$ is a node cover of $G$, then $C[s]:=\cup_{v \in C} v[s]$ is a node cover of $G[s]$. Moreover, every minimal (inclusionwise) node cover of $G[s]$ is of the form $C[s]$ for some (necessarily minimal) node cover $C$ of $G$. In particular, $n c(G[s])=s \cdot n c(G)$.

Definition $2 A$ (nonempty) graph $G=(V, E)$ will be called s-matchable if its s-padding has a perfect matching. A graph $G$ is said to be s-safe if for every node cover $C$ of $G$ the subgraph induced by $C$ is s-matchable.

Remark. Any graph that admits covering of its node set by (pairwise) node disjoint edges and cycles, is 2-matchable. Examples of 2-safe graphs are cliques of size at least three, or cliques of size at least five without an edge. 
Clearly, the definition of an $s$-safe graph makes sense only for even positive integer $s$. For $s$ odd in each graph $G$ there exists a node cover $C$ with odd number of nodes (either $V$, or $V$ without a node). Thus $s$-padding of $C$ has also odd number of nodes and cannot has a perfect matching, which means that the subgraph induced by $C$ is not $s$-matchable.

Theorem 1 Let $s$ be an even positive integer, and $G=(V, E)$ be a graph with the following property: there is a partition $V_{1} \cup V_{2} \cup \cdots \cup V_{p}$ of the node set $V$ such that for each $i \in\{1,2, \ldots, p\}$ the induced subgraph $G_{i}=\left(V_{i}, E_{i}\right)$ of $G$ is s-safe. Then the s-padding of $G$, the graph $G[s]$, has the following property: every minimal node cover of $G[s]$ induces the subgraph of $G[s]$ with a perfect matching. Hence $G[s] \in \mathcal{G}_{0} \subseteq \mathcal{G}$, and eds $(G[s])=\frac{1}{2} n c(G[s])=\frac{s}{2} n c(G)$.

Proof. Consider any minimal node cover of $G[s]$, which is clearly of the form $C[s]$ for some (minimal) node cover $C$ of $G$. The goal is to show that there is a matching $M$ of $G[s]$ with the node set exactly $C[s]$. For each $i=1,2, \ldots, p$, $C \cap V_{i}$ is a node cover of $G_{i}$ (not necessarily minimal). Let $H_{i}$ be the subgraph induced in $G_{i}$ (or, equivalently, in $G$ ) by $C \cap V_{i}$. As $G_{i}$ is $s$-safe, $H_{i}$ is $s$ matchable, i.e. $H_{i}[s]$ has a perfect matching. Take one such perfect matching $M_{i}$ of $H_{i}[s]$ for each $i \in\{1,2, \ldots, p\}$. Then their union $M:=\cup_{i=1}^{p} M_{i}$ is a matching of $G[s]$ with the node set exactly $C[s]$, as required.

The special case of Theorem 1 , when $s=2$ and all graphs $G_{i}(i=1,2, \ldots, p)$ are cliques of size at least 3 , is enough for the proof of the main Theorem 3 of this paper.

\section{General, bounded and dense instances}

In this section we present two approaches how to achieve lower bound for the Minimum Edge Dominating SeT problem (and hence also Minimum MaxiMAL MATChing) for general graphs. The first capitalizes on inapproximability result for linear equations systems. It can be modified either to bounded degree, or to everywhere dense graphs. The second one relates the problem to parameters in PCP characterization of NP class.

\subsection{Reduction from linear equation systems}

Definition 3 MAX-E3-LIN-2 is the following optimization problem: Given a system $I$ of linear equations over $\mathbb{Z}_{2}$, with exactly 3 (distinct) variables in each equation. The goal is to maximize, over all assignments $\varphi$ to the variables, the ratio $\frac{\operatorname{sat}(\varphi)}{|I|}$, where $\operatorname{sat}(\varphi)$ is the number of equations of I satisfied by $\varphi$ and $|I|$ is the cardinality of $I$. Denote Ek-MAX-E3-LIN-2 the restriction of this problem to systems $I$ with exactly $k$ occurrences of each variable used in $I$.

We use the notation $Q(\varepsilon, k)$ for the following partial decision subproblem of MAX-E3-Lin-2: Given an instance of Ek-MAX-E3-LIN-2, decide if the fraction 
of more than $(1-\varepsilon)$ or less than $\left(\frac{1}{2}+\varepsilon\right)$ of all equations is satisfied by the optimal (i.e. maximizing) assignment, under the promise that an input instance has its optimum of one of two types above.

The following theorem follows from Håstad's results [12] and the proof can be found in [4]

Theorem 2 For every $\varepsilon \in\left(0, \frac{1}{4}\right)$ there is a constant $k(\varepsilon)$ such that for every $k \geq k(\varepsilon)$ the partial decision subproblem $Q(\varepsilon, k)$ of MAX-E3-LIN-2 is NP-hard.

Notation. Denote $F(x):=-x \log x-(1-x) \log (1-x), x \in(0,1)$, where $\log$ means the natural logarithm. Further,

$$
G(c, t):=\frac{F(t)+F(c t)}{F(t)-c t F\left(\frac{1}{c}\right)} \quad \text { for } 0<t<\frac{1}{c}<1,
$$

$g(t):=G\left(\frac{1-t}{t}, t\right)$ for $t \in\left(0, \frac{1}{2}\right)$. More explicitly, $g(t)=2 \cdot[-t \log t-(1-$ t) $\log (1-t)] /[-2(1-t) \log (1-t)+(1-2 t) \log (1-2 t)]$. Using Taylor series of the logarithm near 1 we see that the denominator here is $t^{2} \cdot \sum_{k=0}^{\infty} \frac{2^{k+2}-2}{(k+1)(k+2)} t^{k}>t^{2}$, and $-(1-t) \log (1-t)=t-t^{2} \sum_{k=0}^{\infty} \frac{1}{(k+1)(k+2)} t^{k}<t$, consequently $g(t)<$ $\frac{2}{t}\left(1+\log \frac{1}{t}\right)$.

To prove the main Theorem 3 for bounded instances we need some results about regular bipartite expanders.

Definition 4 An $n$ by $n$ bipartite graph $G=(V, E)$ is a $(c, t, d)$-expander if it is d-regular and for each subset $U \subseteq V$ with $|U| \leq$ tn we have $|\Gamma(U)| \geq c|U|$, where $\Gamma(U):=\{y: y$ is a node adjacent to some $x \in U\}$.

In the standard model of random $d$-regular bipartite graphs it is well known that the conditions $0<t<\frac{1}{c}<1$ and $d>G(c, t)$ are sufficient for the existence of a $(c, t, d)$-expander with $n$ by $n$ bipartition for every sufficiently large $n$ (see e.g. Theorem 6.6 in [3]).

An independent set in a graph $G$ is a set of pairwise nonadjacent nodes. The Maximum IndePendent Set problem (shortly, MAX-IS) asks for an independent set of maximum cardinality in $G, \alpha(G)$.

Lemma 1 Let $t \in\left(0, \frac{1}{2}\right)$ and $d$ be an integer such that $d>g(t)$. For every sufficiently large positive integer $n$ there is a d-regular $n$ by $n$ bipartite graph $H$ with bipartition $\left(V_{0}, V_{1}\right)$, such that for each independent set $J$ in $H$ either $\left|J \cap V_{0}\right| \leq t n$, or $\left|J \cap V_{1}\right| \leq t n$.

Proof. If $d>g(t)\left(=G\left(\frac{1-t}{t}, t\right)\right)$, by continuity of the function $G$ also $d>G(c, t)$ for some $c>\frac{1-t}{t}$. For $n$ sufficiently large $(c, t, d)$-expanders exist, as mentioned above, and they clearly have the required property.

In the following we use acronym $B$ in the notation of any graph problem restricted to graphs of degree at most $B$. 
Theorem 3 For every $\delta \in\left(0, \frac{1}{6}\right)$ it is NP-hard to approximate Min-EDS (MinMAXL-MATCH) to within $\frac{7}{6}-\delta$, even in graphs of degree at most $6\left\lfloor g\left(\frac{\delta}{2}\right)\right\rfloor+12 \leq$ $6\left\lceil\frac{4}{\delta}\left(1+\log \frac{2}{\delta}\right)\right\rceil+6$. Consequently, for any $B \geq 462$ it is NP-hard to approximate $B$-Min-Eds (B-Min-MAXL-MATCH) to within any constant smaller than $\frac{7}{6}-$ $\delta(B)$, where $\delta(B):=2 g^{-1}\left(\left\lfloor\frac{B}{6}\right\rfloor-1\right)<\frac{24}{B-12}(\log (B-12)+1-\log 12)<\frac{24 \log B}{B}$.

Proof. (a) First we prove the result for graphs without restriction on degrees. Fix $\delta \in\left(0, \frac{1}{6}\right)$, choose $\varepsilon \in\left(0, \frac{1}{4}\right)$ such that $\frac{7}{6}-\delta<\frac{7-2 \varepsilon}{6+2 \varepsilon}$, and then take $k$ for which $Q(\varepsilon, k)$ is NP-hard. We describe a simple reduction $f$ from E $k$-MAX-E3-LIN-2 to graphs and check how the NP-hard gap of $Q(\varepsilon, k)$ is preserved for the value of eds.

Let $I$ be an instance of E $k$-MAX-E3-Lin-2, $\mathcal{V}(I)$ be the set of variables of $I$, and $m:=|\mathcal{V}(I)|$. Clearly the system $I$ has $\frac{m k}{3}$ equations. For each equation we take simple equation gadget, a clique of size 4 . More precisely, if the equation reads as $x+y+z=j(j \in\{0,1\})$ we take a clique of size 4 whose nodes have labels $x y z=00 j, x y z=01(1-j), x y z=10(1-j)$, and $x y z=11 j$. Notice, that nodes correspond to assignments to variables making the equation satisfied. Now we add an edge for each pair of inconsistently labeled nodes. The pair of nodes is inconsistent if a variable $u \in \mathcal{V}(I)$ exists that is assigned differently in their labels. Let us denote the graph obtained in this way by $G_{I}$, and $f(I):=G_{I}[2]$ its 2-padding.

Clearly $G_{I}$ has $\frac{4}{3} m k$ nodes. By Theorem $1, G_{I}[2] \in \mathcal{G}$, hence $\operatorname{eds}\left(G_{I}[2]\right)=$ $\frac{1}{2} n c\left(G_{I}[2]\right)=n c\left(G_{I}\right)$. Denote by $\alpha\left(G_{I}\right)$ cardinality of the maximum independent set in $G_{I}$. We will show that $\alpha\left(G_{I}\right)=\frac{m k}{3} \cdot \operatorname{OPT}(I)$, where $\operatorname{OPT}(I)$ is the fraction of maximum cardinality of satisfiable equations over all assignments $\varphi: \mathcal{V}(I) \rightarrow\{0,1\}$

Given any assignment $\varphi: \mathcal{V}(I) \rightarrow\{0,1\}$, let $J_{\varphi}$ consist of all nodes whose partial assignment is the restriction of $\varphi . J_{\varphi}$ is an independent set and $\left|J_{\varphi}\right|$ is just the number of equations from $I$ that are satisfied by $\varphi$. Hence $\left|J_{\varphi}\right| \leq$ $\frac{m k}{3} \mathrm{OPT}(I)$ for each assignment. Furthermore, there is an assignment for which the equality holds.

Let us consider now an arbitrary independent set $J$ in $G_{I}$. From the definition of edges in $G_{I}$ it follows that there are no inconsistencies among partial assignments determined by nodes of $J$. Hence there is an assignment $\varphi$ such that $J \subseteq J_{\varphi}$. Now $\alpha\left(G_{I}\right)=\frac{m k}{3} \mathrm{OPT}(I)$ easily follows. Further, $n c\left(G_{I}\right)=\frac{4}{3} m k-\alpha\left(G_{I}\right)=\frac{m k}{3}(4-\mathrm{OPT}(I))$. Hence, the NP-hard question of whether $\operatorname{OPT}(I)$ is greater than $(1-\varepsilon)$, or it is in the interval $\left\langle\frac{1}{2}, \frac{1}{2}+\varepsilon\right)$ is transformed to the NP-hard partial decision problem of whether $n c\left(G_{I}\right)$ $\left(=e d s\left(G_{I}[2]\right)\right.$ is less than $\frac{m k}{3}(3+\varepsilon)$, or it is in the interval $\left(\frac{m k}{3}\left(\frac{7}{2}-\varepsilon\right), \frac{m k}{3} \cdot \frac{7}{2}\right\rangle$. Consequently, it is NP-hard to approximate the solution of both studied problems to within $\frac{7 / 2-\varepsilon}{3+\varepsilon}\left(>\frac{7}{6}-\delta\right)$.

(b) To prove inapproximability within $\frac{7}{6}-\delta$ for bounded degree graphs one can use the idea from [6]: to replace graph $G_{I}$ of all inconsistencies by its lower degree subgraph with suitable expanding properties. 
Let $\delta \in\left(0, \frac{1}{6}\right)$ be given, put $d:=\left\lfloor g\left(\frac{\delta}{2}\right)\right\rfloor+1\left(\leq\left\lceil\frac{4}{\delta}\left(1+\log \frac{2}{\delta}\right)\right\rceil\right)$. As $d>g\left(\frac{\delta}{2}\right)$ we choose $t \in\left(0, \frac{\delta}{2}\right)$ so that $d>g(t)$. Further we take $\varepsilon \in\left(0, \frac{1}{4}\right)$ such that $\frac{7 / 2-\varepsilon-6 t}{3+\varepsilon}>\frac{7}{6}-\delta$. Then a positive integer $k$ is chosen so that (i) $Q(\varepsilon, k)$ is NP-hard (see Theorem 2), and (ii) there is a $d$-regular $2 k$ by $2 k$ bipartite graph $H$ with bipartition $\left(V_{0}, V_{1}\right)$, such that for each independent set $J$ in $H$ either $\left|J \cap V_{0}\right| \leq 2 k t$, or $\left|J \cap V_{1}\right| \leq 2 k t$ (see Lemma 1). Keep one such graph $H$ fixed from now on.

Now we start with an instance $I$ of Ek-MAX-E3-Lin-2, and let $m:=|\mathcal{V}(I)|$. We take the same equation gadget as in part (a). It means a clique of size 4 , with nodes labeled by all 4 satisfying assignments to variables in that equation. Consider a variable $u \in \mathcal{V}(I)$. Let $V_{j}(u)(j \in\{0,1\})$ be the set of all $2 k$ nodes in which $u$ has assigned bit $j$. In the part (a) we created the graph $G_{I}$ such that for each $u \in \mathcal{V}(I)$ the complete bipartite graph with bipartition $\left(V_{0}(u), V_{1}(u)\right)$ is the subgraph of $G_{I}$. Now we create a graph $G_{I}^{H}$ on the same set of nodes as $G_{I}$ (from the part (a)) but with maximum degree at most $3 d+3$, as follows: For each $u \in \mathcal{V}(I)$ we take edges between $V_{0}(u)$ and $V_{1}(u)$ exactly as prescribed by the fixed expander $H$. Having this done one after another, for each $u \in \mathcal{V}(I)$, we get the graph $G_{I}^{H}$. Let $h(I):=G_{I}^{H}[2]$ be its 2-padding.

Clearly, the transformation $h$ is polynomial, $G_{I}^{H}$ is the graph of maximum degree at most $3 d+3$, and $h(I)$ is of maximum degree at most $6 d+6$. Again by Theorem 1, $G_{I}^{H}[2] \in \mathcal{G}$ and hence $\operatorname{eds}\left(G_{I}^{H}[2]\right)=\frac{1}{2} n c\left(G_{I}^{H}[2]\right)=n c\left(G_{I}^{H}\right)$. Clearly, any independent set in $G_{I}$ is also an independent set in $G_{I}^{H}$, hence $\alpha\left(G_{I}^{H}\right) \geq \alpha\left(G_{I}\right)=\frac{m k}{3} \mathrm{OPT}(I)$ and $n c\left(G_{I}^{H}\right) \leq n c\left(G_{I}\right)=\frac{m k}{3}(4-\mathrm{OPT}(I))$.

On the other hand, we can show that $\alpha\left(G_{I}^{H}\right) \leq \alpha\left(G_{I}\right)+2 k m t$ as follows: consider an independent set $J$ of $G_{I}^{H}$ with $|J|=\alpha\left(G_{I}^{H}\right)$. For each $u \in \mathcal{V}(I)$, one after another, remove exactly one of sets $J \cap V_{0}(u), J \cap V_{1}(u)$ from $J$, namely the one with cardinality $\leq 2 k t$. (The existence of such set is ensured by properties of our expander $H$, and the way how $G_{I}^{H}$ was created.) Having this done for all $u \in \mathcal{V}(I)$, we get an independent set of $G_{I}$ (hence of size $\leq \alpha\left(G_{I}\right)$ ) removing no more than $2 k m t$ nodes. Hence

$$
\begin{aligned}
\alpha\left(G_{I}^{H}\right) & \leq \alpha\left(G_{I}\right)+2 k m t=\frac{m k}{3}(\mathrm{OPT}(I)+6 t) \text { and } \\
n c\left(G_{I}^{H}\right) & \geq \frac{m k}{3}(4-\mathrm{OPT}(I)-6 t) .
\end{aligned}
$$

It means, that the NP-hard question of whether $\mathrm{OPT}(I)$ is greater than $(1-\varepsilon)$ or less than $\frac{1}{2}+\varepsilon$, is transformed to the NP-hard partial decision problem of whether $n c\left(G_{I}^{H}\right)\left(=e d s\left(G_{I}^{H}[2]\right)\right)$ is less than $\frac{m k}{3}(3+\varepsilon)$, or greater than $\frac{m k}{3}\left(\frac{7}{2}-\varepsilon-6 t\right)$.

Consequently, it is NP-hard to approximate (on instances $G_{I}^{H}[2]$ of maximal degree $\leq 6 d+6)$ Min-EDS and Min-MAXL-MATCH to within $\frac{7 / 2-\varepsilon-6 t}{3+\varepsilon}>\frac{7}{6}-\delta$.

(c) For large enough $B$ we look for $\delta \in\left(0, \frac{1}{6}\right)$ such that $6\left\lfloor g\left(\frac{\delta}{2}\right)\right\rfloor+12 \leq B$. As $g\left(\frac{1}{12}\right) \approx 75.62$ and $g$ is decreasing in $\left(0, \frac{1}{12}\right\rangle$, we can see that for $B \geq 462$ any $\delta>\delta(B):=2 g^{-1}\left(\left\lfloor\frac{B}{6}\right\rfloor-1\right)$ will do. Trivial estimates on $\delta(B)$ (using $\left.g(t)<\frac{2}{t}\left(1+\log \frac{1}{t}\right)\right)$ are $\delta(B)<\frac{24}{B-12}(\log (B-12)+1-\log 12)<\frac{24 \log B}{B}$. 
Consequences about inapproximability of $B$-Min-Eds (and also B-Min-MaxLМАTCH) are straightforward.

Definition 5 For a constant $\theta \in(0,1)$, everywhere $\theta$-dense graph is a graph $G=(V, E)$ of minimum degree at least $\theta|V|$.

Theorem 4 For any $\theta \in(0,1)$, it is NP-hard to approximate Min-Eds (MinMAXL-MATCH) on everywhere $\theta$-dense graphs to within any constant smaller than $\frac{7+\theta}{6+2 \theta}$.

Proof. Let $\theta \in(0,1)$ be fixed and $r \in\left(1, \frac{7+\theta}{6+2 \theta}\right)$. To prove inapproximability to within $r$ on everywhere $\theta$-dense graphs, we choose $\varepsilon>0$ and $\omega>\frac{\theta}{1-\theta}$ such that $\frac{7-2 \varepsilon+8 \omega}{6+2 \varepsilon+8 \omega}>r$. This is clearly possible by continuity, as for $\varepsilon=0$ and $\omega=\frac{\theta}{1-\theta}$ the last inequality reads as $\frac{7+\theta}{6+2 \theta}>r$.

Now we choose $k$ for which $Q(\varepsilon, k)$ is NP-hard. For any instance $I$ of Ek-MAX-E3-Lin-2 we create the graph $G_{I}$ as in the proof of Theorem 3 (using the same notation). Consider the graph $G_{I}^{\prime}$ obtained from $G_{I}$ by adding a clique with $\left\lfloor\frac{4}{3} m k \omega\right\rfloor$ nodes and connecting every node of the clique to every node of $G_{I}$. It is easy to check that $G_{I}^{\prime}$ and $G_{I}^{\prime}[2]$ are everywhere $\theta$-dense (assuming $m k$ is large enough). By Theorem $1, G_{I}^{\prime}[2] \in \mathcal{G}$, hence eds $\left(G_{I}^{\prime}[2]\right)=n c\left(G_{I}^{\prime}\right)$. Moreover, $n c\left(G_{I}^{\prime}\right)=n c\left(G_{I}\right)+\left\lfloor\frac{4}{3} m k \omega\right\rfloor=\frac{m k}{3}(4-\mathrm{OPT}(I))+\left\lfloor\frac{4}{3} m k \omega\right\rfloor$. Hence, $\operatorname{OPT}(I)>1-\varepsilon$ implies eds $\left(G_{I}^{\prime}[2]\right)<\frac{m k}{6}(6+2 \varepsilon+8 \omega)$, and $\operatorname{OPT}(I)<\frac{1}{2}+\varepsilon$ implies eds $\left(G_{I}^{\prime}[2]\right)>\frac{m k}{6}(7-2 \varepsilon+8 \omega)-1$. Consequently, to approximate eds on $\theta$-dense instances $G_{I}^{\prime}[2]$ to within $r$ is NP-hard.

\subsection{PCP based proof}

We show that the problem MIN-EDS relates in a straightforward way to parameters of PCP systems. Firstly, recall some basic notation for verifiers and the parametric complexity classes.

A verifier $V$ is an oracle probabilistic polynomial-time Turing machine. During its computation, $V$ reads an input, tosses random coins, and has oracle access to a string $\pi$ called proof. A verifier $V$ is called $(r, q)$-restricted if for any input $x$ and for any proof $\pi, V$ generates a random string $R$ tossing $r(x)$ coins and queries to $\pi$ via oracle access $q(x)$ times. Then it outputs $V^{\pi}(x, R) \in\{$ accept $=1$, reject $=0\}$.

Definition 6 A language $L$ belongs to the class $P C P_{c, s}[r, q]$, where $c$, $s$ are completeness and soundness probabilities, if there exists an $(r, q)$-restricted verifier $V$ that given an input $x$ and oracle access to $\pi$ has the following properties:

- for $x \in L$ there is a membership proof $\pi$ such that the verifier accepts $\pi$ with probability $\geq c$;

- for $x \notin L$ and each membership proof $\pi$ the probability that the verifier $V$ accepts $\pi$ is $<$ s. 
The probability is taken over all random strings $R \in\{0,1\}^{r(|x|)}$.

We consider the FGLSS graphs which naturally appear in a general reduction (so called FGLSS-reduction [9]) from languages having efficient PCP (Probabilistic Checking of Proof) systems to approximation versions of MAXIMUM Independent Set (or Maximum Clique) and Minimum Node Cover.

For a verifier $V$ and an input $x$ the graph $G_{x}$ (more precisely $G_{V, x}$ ), the FGLSS graph corresponding to $V$ and $x$, is defined as follows: Every node in $G_{x}$ corresponds to an accepting configuration $(R, Q) \in\{0,1\}^{r(x)} \times\{0,1\}^{q(x)}$ of $V$ 's computation. That means, for each random string $R$ we enumerate the $2^{q(x)}$ possible binary sequences that represent possible sequence of answers to $V$ 's oracle queries. For each such sequence $Q$, we include the pair $(R, Q)$ as a node of $G_{x}$ if $V$ accepts the sequence $Q$ on random string $R$. The edges of $G_{x}$ correspond to inconsistencies among these configurations. That is, there is an edge between $(R, Q)$ and $\left(R^{\prime}, Q^{\prime}\right)$ if there is a query $\pi[i]$ that will be asked by $V$ on both $(x, R)$ and $\left(x, R^{\prime}\right)$, and it has different responses in $Q$ and $Q^{\prime}$.

The accepting configurations of the form $(R, \cdot)$ for a fixed random string $R$ form a layer. Each layer clearly induces a clique in $G_{x}$. A verifier has average free bit complexity $f_{a v}(x)$ if the sum of sizes of layers is $2^{r(x)+f_{a v}(x)}$. Notice, that this is the number of nodes of the graph $G_{x}$.

For application to problems like MIN-NC it is important that $f_{a v}$ is bounded above by small constant, $f_{*}$, independent of $x$. For our application to Min-EDS it is further important that we can work with verifiers for which all layers have size at least 3 . Then due to Theorem 1, 2-padding of $G_{x}$ satisfies $\operatorname{eds}\left(G_{x}[2]\right)=$ $n c\left(G_{x}\right)$.

An independent set of $G_{x}$ corresponds to a proof for $x$ and the size of this set is $2^{r}$ times the probability that $V$ accepts this proof. Thus if $x \in L$ there is an independent set of size $c 2^{r}$ (hence $n c\left(G_{x}\right) \leq 2^{r}\left(2^{f_{a v}}-c\right)$ ), whereas if $x \notin L$ the size of any independent set in $G_{x}$ is less than $s 2^{r}$ (and hence $n c\left(G_{x}\right)>2^{r}\left(2^{f_{a v}}-\right.$ $s))$. As $\frac{2^{f_{a v}-s}}{2^{f_{a v}-c}}=1+\frac{c-s}{2^{f_{a v}-c}} \geq 1+\frac{c-s}{2^{f_{*}-c}}$, any algorithm that approximates $e d s$ (on graphs $G_{x}[2]$ ) to within $1+\frac{c-s}{2_{*} f_{*}}$ would be sufficient to decide if $x \in L$.

The reduction above has polynomial time complexity if $r(x)=O(\log |x|)$ and $q$ is bounded above by a constant. Hence if for some NP-complete language $L$ there is a proof that $L \in \mathrm{PCP}_{c, s}[O(\log |x|), O(1)]$ using verifier $V$ with average free bit complexity $\leq f_{*}\left(f_{*}\right.$ being constant $)$ and with at least 3 accepting configurations for any random string $R$, then approximation of eds to within $1+\frac{c-s}{2^{f_{*}-c}}$ is NP-hard. Applying Håstad's result [12] that for every $\varepsilon \in\left(0, \frac{1}{4}\right)$ $\mathrm{NP} \subseteq \mathrm{PCP}_{1-\varepsilon, 0.5+\varepsilon}[O(\log |x|), 3]$ using verifier with 3 queries and exactly 4 accepting configurations for any random string $R$ (hence $f_{a v}=f_{*}=2$ ), we obtain again that it is NP-hard to approximate MiN-EDS to within any constant smaller than $\frac{7}{6}$. 


\section{Small degree and sparse instances}

For small degree and sparse instances it is more difficult to obtain interesting lower bounds on efficient approximability (unless $\mathrm{P}=\mathrm{NP}$ ). There is no surprise that for these cases also trivial upper bound 2 can be improved. For example, there is a polynomial time approximation algorithm for MiN-EDS on 3-regular graphs with the asymptotic performation ratio of $\frac{3}{2}([8])$.

Theorem 5 It is NP-hard to approximate 3-Min-EDs (and hence 3-MIN-MAXL$\mathrm{MATCH})$ to within any constant smaller than $1+\frac{1}{487}$.

Proof. One of reductions of [18] starts with a 3-regular graph $G$ with $n$ nodes and produces a graph $f(G)$ of maximum degree 3 with $10 n$ nodes and $\frac{21 n}{2}$ edges for which eds $(f(G))=2 n+n c(G)$. Using currently the best inapproximability results for MiN-NC problem on 3-regular graphs ([5]) one can easily find that it is NP-hard to distinguish the case of eds $(f(G))$ being larger than 2.51549586n from that of being smaller than $2.5103305 n$. Hence inapproximability to within $1+\frac{1}{487}$ follows, even on instances produced by $f$.

Slightly better results can be obtained for sparse graphs for which one node is allowed to be of large degree and all the others have small degree. The following simple transformation $g$ from the MiN-NC problem is universal. Given a graph $G=(V, E)$ with $n$ nodes and $m$ edges, add one new special node 0 , connect 0 with every $u \in V$ by an edge, and replace every $e=\{u, v\} \in E$ by a simple gadget $G_{e}$ depicted on the following figure:

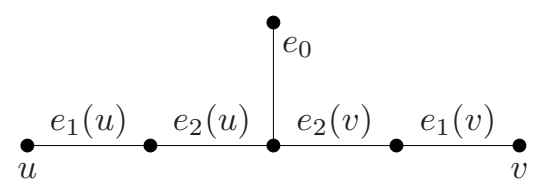

The bipartite graph $g(G)$ constructed in this way has $(n+4 m+1)$ nodes and $n+5 m$ edges. The important fact is that $e d s(g(G))$ is easily related to $n c(G)$.

Lemma 2 eds $(g(G))=m+n c(G)$.

Proof. Consider the class $\mathcal{E}$ of edge dominating sets in $g(G)$ that are related to some node cover $C$ of $G$ as follows: Given a node cover $C$ of $G$ one can create the corresponding edge dominating set $F$ of $g(G)$ of cardinality $m+|C|$ that contains all edges $\{u, 0\}, u \in C$, and for every $e=\{u, v\} \in E$ one edge from the gadget $G_{e}$, namely $e_{2}(u)$ or $e_{2}(v)$. More precisely, for the edge $e=\{u, v\}$ with $u \notin C$ we take $e_{2}(u)$; for the edge $e=\{u, v\}$ with both nodes $u, v$ in $C$ the choice of either $e_{2}(u)$ or $e_{2}(v)$ can be made arbitrarily. Easily, $F$ is an edge dominating set of $g(G)$. Taking $C$ optimally, i.e. with $|C|=n c(G)$, we get $e d s(g(G)) \leq m+n c(G)$.

To show the opposite inequality, consider any edge dominating set $F$ of $g(G)$ and the goal is to prove that $|F| \geq m+n c(G)$. We will show that $F$ can be 
transformed, without increasing its size, into another edge dominating set $F^{\prime}$ of $g(G)$ such that $F^{\prime} \in \mathcal{E}$.

Firstly, every $e_{0}$ edge (for $e=\{u, v\} \in E$ ) in $F$ can be replaced by $e_{2}(u$ ) or $e_{2}(v)$ (arbitrarily), or possibly removed. Hence we can assume that $F$ does not contain such edges.

Consider now any $e=\{u, v\} \in E$.

(a) Assume first $e_{2}(u) \in F$. If $F \cap\left\{e_{1}(v), e_{2}(v)\right\} \neq \emptyset$, replace it in $F$ by an edge $\{v, 0\}$. If $e_{1}(u) \in F$, replace it by $\{u, 0\}$. We will end with an edge dominating set containing exactly one edge from $G_{e}$, the edge $e_{2}(u)$.

(b) Assume now $e_{2}(u) \notin F$. As $e_{0}(\notin F)$ is dominated by $F, e_{2}(v) \in F$ follows. Now we do the same as in (a) with the role of $u$ and $v$ interchanged. Having this done for each $e=\{u, v\} \in E$, one after another, we will obtain $F^{\prime}$ with one edge from each gadget $G_{e}$ (either $e_{2}(u)$ or $e_{2}(v)$ ) and some edges of the kind $\{u, 0\}, u \in V$. To see that $C:=\left\{u:\{u, 0\} \in F^{\prime}\right\}$ is a node cover of $G$ is easy. If for $e=\{u, v\} \in E$ we have $e_{2}(u) \in F^{\prime}$, clearly $v \in C$ (otherwise $e_{1}(v)$ would not be dominated by $F^{\prime}$ ), if $e_{2}(v) \in F^{\prime}, u \in C$. Hence $F^{\prime}$ of cardinality $m+|C|$ shows that $|F| \geq\left|F^{\prime}\right|=m+|C| \geq m+n c(G)$ that completes the proof.

Theorem 6 It is NP-hard to approximate MiN-EDs (and hence Min-MAXL$\mathrm{MATCH)} \mathrm{for} \mathrm{bipartite} \mathrm{graphs} \mathrm{with} \mathrm{all} \mathrm{nodes} \mathrm{but} \mathrm{one} \mathrm{of} \mathrm{degree} \mathrm{at} \mathrm{most} 4$ (resp. 5) to within any constant smaller than $1+\frac{1}{390}$ (resp., $\left.1+\frac{1}{250}\right)$.

Proof. Using the reduction $g$ above, these results easily follow from known hardness results for bounded instances of the Minimum Node Cover problem $([5])$. For a 3-regular graph $G$ with $n$ nodes the bipartite graph $g(G)$ has $7 n+1$ nodes, $\frac{17}{2} n$ edges, and all nodes but one of degree $\leq 4$.

Applying the NP-hard gap results of [5] for the Minimum Node Cover problem in 3-regular graphs, the corresponding NP-hard question is now to decide of whether $\operatorname{eds}(g(G))$ is larger than 2.01549586n, or smaller than 2.0103305n. Hence to approximate $e d s$ on such instances to within $1+\frac{1}{390}$ is NP-hard.

The lower bound is slightly better if we start with 4-regular graphs. For a 4-regular graph $G$ with $n$ nodes the bipartite graph $g(G)$ has $9 n+1$ nodes, $11 n$ edges, and all nodes but one of degree $\leq 5$. Now it is NP-hard to decide of whether eds $(g(G))$ is larger than 2.53036437246n, or smaller than 2.52024291497n. Hence to approximate eds on such instances to within $1+\frac{1}{250}$ is NP-hard.

\section{Other similar problems}

Definition 7 If $G=(V, E)$ is a graph then the total graph of $G$, denoted by $T(G)$, is defined as $T(G)=\left(V \cup E, E \cup E^{\prime} \cup E^{\prime \prime}\right)$, where $E^{\prime}=\{\{e, v\}: e \in E$, $v \in V$ and $v$ is incident with $e\}$, and $E^{\prime \prime}=\{\{e, f\}: e, f \in E$ are adjacent edges\}. 
In the following theorem we present lower bound on approximability for some graph optimization problems in total graphs. Recall that for given $T(G)$ one can reconstruct $G$ in polynomial time, see [11].

Theorem 7 In the class of total graphs it is NP-hard to approximate the MAXIMUM INDEPENDENT SET problem to within any constant smaller than $\frac{10}{9}$, and the Minimum Node Cover problem, Min-Eds, Min-Maxl-Match to within any constant smaller than $1+\frac{1}{1336}$.

Proof. One can prove that $\alpha(T(G))=|V(G)|-e d s(G)$ (see, e.g. [18]). In the proof of Theorem 3 we produced instances $G=(V, E)$ with $n:=\frac{8}{3} m k$ nodes for which it was NP-hard to distinguish between the case of $e d s(G)<\frac{n}{16}(6+2 \varepsilon)$ and the one of $e d s(G)>\frac{n}{16}(7-2 \varepsilon)$. For the problem of Maximum InDEPENDENT Set in total graphs $T(G)$ (known as the Maximum Total Matching problem for the original graph $G$ ) two cases above translate as $\alpha(T(G))>\frac{n}{16}(10-2 \varepsilon)$ and $\alpha(T(G))<\frac{n}{16}(9+2 \varepsilon)$, respectively. Hence it is NP-hard to approximate the Maximum Total Matching problem to within any constant smaller than $\frac{10}{9}$. On the other hand, it is easy to design $\frac{3}{2}$-approximation algorithm for the Maximum Total Matching problem. It suffices to find any maximal matching $M$ of $G$ and return $M \cup(V \backslash V(M))$; it is an independent set in $T(G)$ of size at least $\frac{2}{3} \alpha(T(G))$.

Passing to the complementary problem Min-NC one gets $n c(T(G))=|E(G)|+$ $e d s(G)$. To obtain an interesting explicit lower bound on approximability of MiN-NC in total graphs, one can use the NP-hard gap result for Min-EDS in sparse graphs. The NP-hard gap of MiN-EDS for sparse graphs from Lemma 6 (all nodes but one of degree $\leq 5$ ) transforms to the one showing that to approximate Min-NC in total graphs within $1+\frac{1}{1336}$ is NP-hard. The NP-hard gap with the same inapproximability applies to MiN-Eds (Min-MaXL-Match) in total graphs as well. This is due to the fact that in $T(G)$ any node cover with even number of nodes induces the graph with a perfect matching, assuming that $G$ was connected (see, e.g., [13]). It implies that for a connected graph $G$, $e d s(T(G))=\left\lceil\frac{n c(T(G))}{2}\right\rceil$.

Having NP-hard gap result for MiN-EDS in total graphs, we can use the previous result for the graph $T(G)$ in place of $G$ to show the NP-hard gap result for MAX-IS of 2-iterated total graph of $G, T(T(G))$.

Using mathematical induction, for any positive integer $r$ we can derive explicit NP-hard gap result for each of problems MaX-IS, Min-NC, Min-Eds, Min-MaXl-Match restricted to the $r$-iterated total graphs.

The fact, that the lower bounds for polynomial time approximability of these problems converge very rapidly to 1 with increasing $r$, does not necessarily mean that those results are weak. In fact, one can show the upper bounds of the form $1+\delta^{r^{2}}$, for some constant $\delta \in(0,1)$, for these problems on the $r$-iterated total graphs.

Remark. Min-Eds is equivalent to the Minimum (Node) Dominating Set problem (Min-DS) restricted to line graphs. Hence this restricted version of 
Min-DS is APX-complete, has simple 2-approximation algorithm, but it is NPhard to approximate to within $\frac{7}{6}-\delta$ for any $\delta>0$. Let us mention that for general graphs Min-DS is not in APX; it is as hard to approximate as the set cover problem.

\section{References}

[1] B. S. Baker: Approximation algorithms for NP-complete problems on planar graphs, Journal of ACM 41(1994), 153-180.

[2] R. Carr, T. Fujito, G. Konjevod and O. Parekh: A $2 \frac{1}{10}$-approximation algorithm for a generalization of the weighted edge-dominating set problem, Journal of Combinatorial Optimization 5(2001), 317-326.

[3] F. R. K. Chung: Spectral Graph Theory, CBMS Regional Conference Series in Mathematics, American Mathematical Society, 1997, ISSN 01607642, ISBN 0-8218-0315-8.

[4] M. Chlebík and J. Chlebíková: Approximation hardness for small occurrence instances of NP-hard problems, Proc. of the 5th CIAC, LNCS 2653, 2003, Springer, 152-164 (also ECCC Report TR02-73, 2002).

[5] M. Chlebík and J. Chlebíková: Inapproximability results for bounded variants of optimization problems, Proc. of the 14th Inter. Symp. on Fundamentals of Computation Theory, FCT 2003, Malmö, Sweden, August 1215, 2003, LNCS 2751, 2003, Springer, 27-38 (also ECCC Report TR03-26, 2003).

[6] A. Clementi and L. Trevisan: Improved non-approximability results for minimum vertex cover with density constraints, Theor. Computer Science 225(1999), 113-128.

[7] I. Dinur and S. Safra: The importance of being biased, Proc. of the 34th ACM Symposium on Theory of Computing, STOC 2002, 33-42.

[8] W. Duckworth and N. C. Wormald: Linear Programming and the WorstCase Analysis of Greedy Algorithms on Cubic Graphs, preprint.

[9] U. Feige, S. Goldwasser, L. Lovász, S. Safra and M. Szegedy: Approximating clique is almost NP-complete. Proc. of the 32nd IEEE Symposium on Foundations of Computer Science, pp. 2-12, 1991.

[10] T. Fujito and H. Nagamochi: A 2-approximation algorithm for the minimum weight edge dominating set problem, Discrete Appl. Math. 118(2002), 199-207.

[11] F. Gavril: A recognition algorithms for the total graph, Networks 8(1978), 121-133. 
[12] J. Håstad: Some optimal inapproximability results, Journal of ACM 48(2001), 798-859.

[13] J. D. Horton and K. Kilakos: Minimum edge dominating sets, SIAM J. Discrete Math. 6(1993), 375-387.

[14] H. B. Hunt III, M. V. Marathe, V. Radhakrishnan, S. S. Ravi, D. J. Rosenkrantz and R. E. Stearns: A unified approach to approximation schemes for NP- and PSPACE-hard problems for geometric graphs, Proc. of the 2nd Annual European Symposium on Algorithms, ESA 1994, LNCS 855, 1994, 424-435.

[15] S. Mitchell and S. Hedetniemi: Edge domination in trees, Proc. of the 8th Southearn Conference on Combinatorics, Graph Theory, and Computing, 1977, 489-509.

[16] O. Parekh: Edge dominating and hypomatchable sets, Proc. of the 13th Annual ACM-SIAM Symposium on Discrete Algorithms, SODA 2002, pp. 287-291.

[17] A. Srinivasan, K. Madhukar, P. Nagavamsi, C. Pandu Rangan and M.-S. Chang: Edge domination on bipartite permutation graphs and cotriangulated graphs, Inf. Proc. Letters 56(1995), 165-171.

[18] M. Yannakakis and F. Gavril: Edge dominating sets in graphs, SIAM J. Appl. Math. 38(1980), 364-372. 